\title{
A New Syndrome of Axial Muscle Rigidity Associated with Etretinate Therapy ${ }^{1}$
}

\author{
*Roger L. Albin, †Alan K. Silverman, †Charles N. Ellis, \\ †John J. Voorhees, and *James W. Albers \\ Departments of ${ }^{*}$ Neurology and †Dermatology, University of Michigan Medical Center, \\ Ann Arbor, Michigan, U.S.A.
}

\begin{abstract}
Summary: We report on three cases of a novel syndrome associated with treatment for psoriasis with the retinoid etretinate. The distinctive features of this syndrome include rigidity that is restricted to the axial muscles, impairment of neck extension greater than flexion, impairment of anteflexion at the waist, severe impairment of lateral flexion at both the waist and the neck, and, in one case, rigidity of proximal lower extremity muscles. Peripheral nerve block extinguished the lower extremity rigidity in one patient, and two patients had a favorable response to Sinemet. One patient had persistence of symptoms and findings 2 years after discontinuation of etretinate. The clinical features of this syndrome are consistent with an effect of etretinate on central, possible catecholaminergic neurons. Key Words: Etretinate-Retinoid-Rigidity.
\end{abstract}

Etretinate (Etr) (Tegison) is an aromatic vitamin A derivative that has proved to be effective in the treatment of psoriasis and other cutaneous disorders (1). The common side effects of Etr therapy consist of skin and mucosal xerosis, alopecia, arthralgias, myalgias, hyperostoses, and ligamentous calcification (1-3). In contrast to the hypervitaminosis A syndrome, neurologic toxicities, such as headache, dizziness, and increased intracranial pressure, are said to be unusual (4). However, sensory peripheral neuropathy and sleep disorder have been associated with Etr use (ref. 5 and Aldrich M, personal communication).

We have previously reported a patient with a distinctive syndrome of axial muscle rigidity associated with Etr therapy (6). We now report further follow-up on this case (case 1, see below) and two additional cases.

\footnotetext{
1 A videotape segment accompanies this article.

Address correspondence and reprint requests to Dr. Roger L. Albin, Neuroscience Building, 1103 E. Huron Street, Ann Arbor, MI 48104, U.S.A.
} 


\section{Case 1}

A 40-year-old white male with severe psoriasis of 20 years duration had been unsuccessfully treated with conventional therapy and began Etr in July of 1981 . He received an initial 6 month course of Etr and three subsequent 9 month courses. There was a 3 month drug-free interval between each course. His Etr doses ranged from 0.6 to $1.0 \mathrm{mg} / \mathrm{kg} /$ day and he was not taking any other medications.

While taking Etr his psoriasis improved dramatically, but relapsed within a few weeks of each discontinuation of Etr. Cutaneous and mucosal side effects were observed during each course of therapy.

Within 2-3 months after the initiation of Etr therapy, the patient noted painless muscle stiffness without cramping involving his back, neck, and proximal lower extremities. He complained that his gait had altered; that he tended to walk stooped forward; that he was unable to run; that he had difficulty bending forward from the waist; that extension of his neck was limited. He found symptoms to be limiting in certain respects; he had considerable difficulty in putting on his socks and shoes, and had to have his wife tie his shoelaces. In addition, he was unable to play tennis because his diminished neck extension precluded the visualization of the tossed ball during serving.

The intensity of the stiffness symptoms worsened with each course of Etr, although they remitted within a few weeks of the cessation of the first three courses of Etr therapy.

Neurologic evaluation was initially performed during a third course of Etr therapy. His general physical examination, mental status examination, and cranial nerve function were normal. On motor examination, his power was normal in all extremities, but tone was markedly increased in the proximal lower extremities. The tone abnormality had a plastic quality, and following passive or voluntary flexion of the leg at the hip, the patient's limb would slowly (over seconds) return to the resting position. Gait was broad-based and slow, with flexion at the waist, knees, and neck. When walking, he moved as if his trunk were a rigid object. Associated movements were of normal amplitude and character. Percussion myotonia was absent. Finger-to-nose test, heel-knee-shin test, and rapid rhythmic repetitive movements were normal. Postural stability was unimpaired. Sensory examination was normal to pin-prick, light touch, position, vibration, and cortical sensory functions. Muscle stretch reflexes were normal. The patient had limited extension of his neck and anteflexion at the waist. He was unable to move his head from side to side, and was unable to flex laterally at the waist. Firm paraspinal muscles were noted.

Review of serial spine and hip radiographs obtained during the course of Etr therapy revealed only small bone protuberances arising from several lumbar vertebral bodies.

Nerve conduction and electromyographic studies were normal, with the exception of persistent motor unit activation in the quadriceps muscle during attempted relaxation. As the limb came to rest, all motor units eventually disappeared. 
A percutaneous femoral nerve block was performed under local anesthesia, resulting in flaccid paralysis of the quadriceps muscles.

Two months after the completion of his third course of Etr, neurologic examination and electromyogram were normal. His stiffness recurred within a few weeks after beginning his fourth course of Etr. He was treated with Baclofen; at a dose of $20 \mathrm{mg}$ p.o. t.i.d., both subjective and objective clinical improvement in his lower extremity rigidity were noted. Unfortunately, despite doses as high as $90 \mathrm{mg} /$ day of Baclofen, he continued to have stiffness of his neck and back.

Because of his axial rigidity, Etr was discontinued. However, he continued to suffer from neck and back stiffness even though his proximal lower extremity stiffness resolved (see video segment 1). Despite intensive physical therapy, 2 years after the discontinuation of Etr he was still unable to run, had great difficulty in bending over, and was unable to tie his shoelaces or put on his socks. Examination at that time revealed the same stooped posture and gait, the same impairment of waist anteflexion, the same inability to laterally flex at the waist or neck, and persistently firm paraspinal muscles.

At this point the patient was begun on Sinemet $25 / 100$ three times per day. Within a few weeks of initiating Sinemet therapy, he noted a significant improvement. His subjective sense of stiffness lessened, and he was able to resume running, playing tennis, and putting on his socks.

Examination while on Sinemet showed improvement in posture, neck extension, ability to bend forward, and lateral flexion at the waist. He was again able to run (see video segment 2 ).

\section{Case 2}

This patient is a 60 -year-old white male who had severe psoriasis when begun on Etr in 1979. He underwent several courses of Etr with doses ranging from 0.5 to $1.0 \mathrm{mg} / \mathrm{kg} / \mathrm{day}$, and had an excellent response with remission of his psoriasis when taking Etr. Within a few weeks of initiating Etr, he noted low back, neck, and hip stiffness. This was accompanied by some achy discomfort in the affected areas. These complaints persisted over the subsequent years, although the patient noted improvement in his stiffness symptoms whenever he went on a drug holiday. Like patient 1 , he also noted difficulties with bending and lateral flexion. He had particular difficulty with tying his shoes and putting on his socks. He also noted that he had developed a somewhat stooped posture and that his gait speed had diminished. On initial examination in 1984, he had a stooped, flexed posture, impaired bending at the waist, inability to flex laterally at the waist or neck, and tense paraspinal muscles. When walking, he moved as if his trunk were a rigid object. Lower extremity tone was normal and no other abnormalities were noted. Electromyography revealed persistent activation of motor units during attempted relaxation. Plain radiographs of the spine obtained in 1985 revealed multiple hyperostoses in cervical, thoracic, and lumbar spine without evidence of progression from prior films of 1983. 
When re-examined 3 months after discontinuation of Etr he was found to be subjectively and objectively improved. His back stiffness had improved, he was able to bend further forward, and he had recovered his ability to flex laterally at the waist and neck.

Due to worsening of his psoriasis, he resumed use of Etr. His axial rigidity worsened. He had an equivocal response to Baclofen (maximum dose $40 \mathrm{mg}$ / day), and subjective but not objective improvement was obtained with Valium at a dose of $5 \mathrm{mg}$ p.o. t.i.d.

A trial of Sinemet $25 / 100$ four times per day was initiated. Within 2 to 3 weeks of beginning Sinemet he noted symptomatic relief. Examination while on Sinemet revealed clear evidence of improvement. His posture had become upright, his gait speed had increased, his ability to bend forward improved, as did his lateral flexion.

\section{Case 3}

This patient is a 59-year-old white male with severe psoriasis refractory to conventional treatment. The patient was initially treated with Etr in 1979. His doses ranged from 0.3 to $1.0 \mathrm{mg} / \mathrm{kg} / \mathrm{day}$. During several courses of Etr therapy he reported the development of a stiff back with prominent difficulties in bending at the waist. These problems occurred only when the patient received from 0.5 to $1.0 \mathrm{mg} / \mathrm{kg} /$ day of Etr. When the dose was reduced or discontinued, his symptoms remitted. Plain radiographs of the spine obtained in 1984 and 1985 revealed parasyndesmophytes in thoracic and lumbar spine, and minimal findings of idiopathic skeletal hyperostosis.

The patient was initially examined in 1984 during a period of drug holiday. At that time he was symptom-free, and neurologic examination was normal. When re-examined several months later he was taking $0.6 \mathrm{mg} / \mathrm{kg} / \mathrm{day}$ of Etr and was complaining of a recurrence of his stiffness symptoms. At that time he was noted to have a stooped posture, stiff paraspinal muscles, impaired forward flexion at the waist, and absent lateral flexion at the waist and neck. A therapeutic trial of Valium produced little benefit. He has not sought further treatment for his symptoms.

\section{DISCUSSION}

We describe three patients with a unique clinical picture of axial muscle rigidity associated with Etr therapy for psoriasis. Two of these patients were treated with Sinemet and had improvement of their rigidity.

Because of the unusual symptoms and clinical findings seen in these cases, the anatomic localization and pathophysiology of this syndrome is unclear. The results of the percutaneous nerve block performed in case 1 argue that this is a neurogenic, as opposed to myogenic, articular, or skeletal process. The lack of abnormalities (other than an abnormal relaxation pattern) on electroneuromyography not only argues against disease of muscle, but is also against disorders of peripheral nerve, root, or anterior horn cell. By exclusion, the likely locus of 
pathology is within the central nervous system. This conclusion is supported by the favorable response to Sinemet (patients 1 and 2) and Baclofen (patient 1). The absence of cranial nerve abnormalities suggests a spinal cord localization.

This syndrome is easily distinguished from extrapyramidal disorders causing rigidity by the axial distribution of the rigidity and the lack of other findings typically seen in basal ganglia disorders. These include progressive supranuclear palsy where the so-called axial dystonia is usually marked by neck extension (7), not flexion as seen in these cases.

Similarly, this syndrome differs from the stiff-man syndrome (8) and progressive encephalomyelitis with rigidity (9) in that extremities were largely spared, intermittant spasms were absent, the rigidity was not stimulus evoked, and electromyographically abnormality was restricted to an abnormal relaxation pattern. In addition, the stiff-man syndrome has been reported to worsen with L-Dopa administration (10) and improve with clonidine (11), an alpha-2 agonist which reduces catecholamine release.

The only similar cases we have been able to discover in the literature are those of Leigh et al. (12) and Alberca et al. (13). They described individuals with reflex myoclonus and severe abdominal, paraspinal, and lower extremity rigidity. Their patients had a favorable response to diazepam. The patient of Leigh et al. also had nystagmus, cerebellar atrophy on pneumoencephalography, and electrophysiologic studies suggesting a medullary origin for the myoclonus. Leigh et al. concluded that their patient's syndrome was the result of a brainstem abnormality.

The favorable response to Sinemet in two of our patients suggests a deficiency of dopamine or another catecholamine. This could not be a major impairment of the nigrostriatal projection as none of these patients had features of parkinsonism. Specifically, there were no abnormalities of ocular motility, associated movements were normal, facial hypominia was absent, distal limb tone was normal, and bradykinesia and tremor were absent. An alternative localization would be an impairment of descending catecholaminergic tracts innervating the spinal cord. These include dopaminergic, nonadrenergic, and adrenergic projections that originate in the hypothalamus, pons, and medulla $(14,15)$. These projections may be important in nociception, reflex modulation, and the control of ambulation (15).

With respect to pathogenesis, the history of Case 1 suggests either very slow elimination of Etr or a toxic effect of Etr on central neurons. Etr has a serum half-life of approximately 100 days after chronic administration (16), and Etr can be detected in serum 2 years after stopping treatment (17). It is also conceivable that chronic Etr exposure may impair the function of specific group(s) of CNS neurons in a manner analogous to the effects of MPTP on the substantia nigra. A "stiff gait" was reported in one study of chronic Etr toxicity in dogs. Unfortunately, no further follow-up or CNS pathology was reported (18).

While vitamin $\mathrm{A}$ is not known to play a physiological role in the mature CNS, a potential substrate for retinoid effect on brain cells is evidenced by the presence of cytosolic retinoid receptors in rat brain homogenates (19) and the high concentration of the transport protein, transthyretin, in cerebrospinal fluid (20). 
The axial rigidity syndrome described above may not be solely associated with Etr. In their series of patients suffering from vitamin A intoxication, Muenter et al. (21) reported a patient who had originally been thought to have McArdle's syndrome. Muscle stiffness and cramps are common manifestations of this type of glycogenosis, and it is conceivable that the patient of Muenter et al. could have had a syndrome similar to the one we describe here.

In conclusion, we have described a novel syndrome of axial muscle rigidity associated with Etr therapy. This syndrome appears to be of central nervous system origin and may involve a catecholaminergic pathway. The history of one of these patients suggests some form of persistent effect of Etr on the central nervous system.

Acknowledgment: Supported in part by the Babcock Dermatologic Endowment and Hoffman-La Roche, Inc.

\section{LEGENDS TO VIDEOTAPE}

SEGMENT 1. These segments were recorded in December of 1984, 2 months after the discontinuation of etretinate. Despite $80 \mathrm{mg}$ per day of Baclofen, he continued to experience axial muscle rigidity.

SEGMENT 2. This segment was recorded in March of 1987. The patient had begun Sinemet (25/100 po t.i.d.) 2 months earlier, with improvement in some of his symptoms and findings.

\section{REFERENCES}

1. Ellis CN, Voorhees JJ. Etretinate therapy. J Am Acad Dermatol 1987;16:267-91.

2. Windhorst DB, Nigra T. General clinical toxicology of oral retinoids. J Am Acad Dermatol 1982;6:675-82.

3. DiGiovanna JJ, Helfgott RK, Gerber LH, Peck GL. Extraspinal tendon and ligament calcification associated with etretinate. $N$ Engl J Med 1986;315:1177-1182.

4. Lassus A. Systemic treatment of psoriasis with an oral retinoic acid derivative (RO-9359). Br J Dermatol 1980;102:195-202.

5. Danon MJ, Carpenter S, Weiss V, West D, Garvin JS. Sensory neuropathy associated with longterm etretinate therapy. Neurology 1986;36(Suppl 1):321.

6. Ellis CN, Gilbert M, Cohen KA, et al. Increased muscle tone during etretinate therapy. $J \mathrm{Am}$ Acad Dermatol 1986;14:907-9.

7. Jankovic J. Progressive supranuclear palsy. Neurol Clin N Am 1984;2:473-86.

8. Gordone EE, Janusko DM, Kaufman L. A critical review of the stiffman syndrome. Am J Med 1967;42:582-99.

9. Whiteley AM, Swash M, Urich H. Progressive encephalomyelitis with rigidity. Brain 1976;99:2742.

10. Guilleminault C, Sigwald J, Castaigne P. Sleep studies and therapeutic trial with L-Dopa in a case of stiffman syndrome. Eur Neurol 1973;10:89-96.

11. Meinck HM, Ricker K, Conrad B. The stiff-man syndrome: new pathophysiological aspects from abnormal exteroceptive reflexes and the response to clomipramine, clonidine, and tizanidine. $J$ Neurol Neurosurg Psych 1984;7:280-7.

12. Leigh PN, Rothwell JC, Traub $M$, Marsden $C D$. A patient with reflex myoclonus and muscle rigidity: “jerking stiff-man syndrome." J Neurol Neurosurg Psych 1980;43:1125-31.

13. Alberca R, Romero M, Chaparno J. Jerking stiff-man syndrome. J Neurol Neurosurg Psych 1982;45:1159-60.

14. Lindavall O, Bjorklund A, Skagerberg G. Dopamine-containing neurons in the spinal cord: anatomy and some functional aspects. Ann Neurol 1983;14:255-60. 
15. Marshall KC. Catecholamines and their actions in the spinal cord. In: Davidoff RA, ed, Handbook of the spinal cord, vol. 1. New York: Marcel Dekker, 1983;275-328.

16. Brazzell RK, Colburn WA. Pharmacokinetics of the retinoids isotretinoin and etretinate. $J A m$ Acad Dermatol 1982;6:643-51.

17. DiGiovanna JJ, Zech LA, Ruddel ME, et al. Etretinate: persistent serum levels of a potent teratogen. J Invest Dermatol 1984;82:434.

18. Kamm JJ, Ashenfelter KP, Ehmann CW. Preclinical and clinial toxicology of selected retinoids. In: Sporn MB, Roberts AB, Goodman DS, eds, The Retinoids: Volume 2. Orlando, FL: Academic Press, 1984;288-326.

19. Ong DE, Crow A, Chytil F. Radioimmunochemical determination of cellular retinol- and retinoic acid-binding proteins in cytosols of rat tissues. $J$ Biol Chem 1982;257:13385-9.

20. Herbert J, Wilcox JN, Kim-Thu CP, et al. Transthyretin: A choroid plexus-specific transport protein in human brain. Neurology 1986;36:900-11.

21. Muenter DM, Perry HO, Ludwig J. Chronic vitamin A intoxication in adults. Am J Med $1971 ; 50: 129-36$. 